\title{
Monitoramento e projeções das metas de fatores de risco e proteção para o enfrentamento das doenças crônicas não transmissíveis nas capitais brasileiras
}

\author{
Monitoring and projection of targets for risk and protection factors \\ for coping with noncommunicable diseases in Brazilian capitals
}

Alanna Gomes da Silva (https://orcid.org/0000-0003-2587-5658) ${ }^{1}$

Renato Azeredo Teixeira (https://orcid.org/0000-0002-1259-6812) ${ }^{2}$

Elton Junio Sady Prates (https://orcid.org/0000-0002-5049-186X) ${ }^{1}$

Deborah Carvalho Malta (https://orcid.org/0000-0002-8214-5734) ${ }^{1}$

${ }^{1}$ Escola de Enfermagem, Universidade Federal de Minas Gerais (UFMG). Av Professor Alfredo Balena 190, Santa Efigênia. 30130 100 Belo Horizonte MG Brasil. alannagomessilva@ gmail.com

${ }^{2}$ Faculdade de Medicina, UFMG. Belo Horizonte MG Brasil.

\begin{abstract}
This study aimed to monitor the trends and projections of targets of risk and protection factors for coping with noncommunicable diseases in Brazilian capitals and verify whether the economic crisis and austerity policies have interfered with these targets' behavior. This is a time-series study with data from the Surveillance System for Risk and Protective Factors for Chronic Diseases by Telephone Survey. We analyzed the trends in the prevalence of tobacco use, obesity, physical activity, consumption of fruits and vegetables, and alcohol abuse, and their projections until 2025. The Prais-Winsten regression was employed. We adopted the Interrupted Time-Series, considering the 2006-2014 and 2015-2019 periods. A reduction in tobacco use, increase in obesity, consumption of fruits and vegetables, physical activity, and alcohol use was observed between 2006 and 2014. Most indicators have shown worse performance since 2015. Projections foresee that targets for curbing obesity and alcohol abuse will not be achieved. Some changes were identified in the indicators profiles, reinforcing the importance of the continuous monitoring and sustainability of actions, policies, and programs to promote health and control these diseases and their risk factors.
\end{abstract}

Key words Noncommunicable diseases, Risk factors, Protective factors, Interrupted time-series analysis, Economic recession
Resumo Monitorar as tendências e as projeções das metas de fatores de risco e proteção para o enfrentamento das doenças crônicas não transmissiveis nas capitais brasileiras e verificar se a crise econômica e as politicas de austeridade interferiram no comportamento dessas metas. Estudo de série temporal com dados do Sistema de Vigilância de Fatores de Risco e Proteção para Doenças Crônicas por Inquérito Telefônico. Foram analisadas as tendências das prevalências de fumantes, obesidade, prática de atividade física, consumo de frutas e hortaliças e de bebidas alcoólicas, e suas projeções até 2025. Empregou-se a regressão de Prais-Winsten. Utilizou-se a Série Temporal Interrompida de 2006 a 2014 e 2015 a 2019. Entre 2006 e 2014, houve redução de fumantes e aumento da obesidade, do consumo de frutas e hortaliças, de atividade física e do uso de álcool. A maioria dos indicadores demonstrou pior desempenho a partir de 2015. Pelas projeções, as metas de deter a obesidade e reduzir o uso de álcool não seriam atingidas. Houve mudanças no comportamento dos indicadores, o que reforça a importância do monitoramento contínuo, e da sustentabilidade das ações, políticas e programas de promoção a saúde e de controle dessas doenças e seus fatores de risco.

Palavras-chave Doenças não transmissiveis, $\mathrm{Fa}$ tores de risco, Fatores de proteção, Análise de séries temporais interrompida, Recessão econômica 


\section{Introdução}

As doenças crônicas não transmissíveis (DCNT) são as principais causas de morte global, além de ocasionar mortalidade prematura, incapacidade, perda da qualidade de vida, redução da produtividade e impactos financeiros às famílias, comunidades e sociedade ${ }^{1}$.

Estima-se que anualmente as DCNT sejam responsáveis por $71 \%$ da mortalidade no mundo, o que representa 41 milhões de óbitos ${ }^{2}$. Destes, 15 milhões são prematuros ( $<70$ anos) e cerca de 12 milhões ocorrem em países de baixa e média ren$\mathrm{da}^{2}$. Nas Américas, as DCNT causam 5,5 milhões de óbitos por ano, representando $80,7 \%$ de todas as mortes e, destas, $38,9 \%$ são prematuras ${ }^{3}$. No Brasil, o cenário é semelhante, correspondendo a $74 \%$ da mortalidade geral ${ }^{3}$.

O aumento da carga das DCNT está relacionado com o crescimento dos fatores de risco (FR) modificáveis ${ }^{4}$, como tabagismo, álcool, obesidade, inatividade física e alimentação inadequada ${ }^{1}$. Soma-se, ainda, a importância dos determinantes sociais, especialmente o baixo nível socioeconômico, como um forte preditor de causalidade, morbidade e mortalidade prematura por DCNT, e os efeitos de crises econômicas e políticas de austeridade que afetam as políticas sociais, a qualidade dos serviços, os indicadores e o sistema de saúde, e que aumentam o desemprego, a pobreza e as desigualdades ${ }^{5-7}$.

As DCNT são passíveis de prevenção e as estratégias incluem ações de promoção à saúde, adoção de estilos de vida saudáveis e estímulo aos fatores protetores, como a alimentação saudável e a atividade física. Nesse sentido, o Ministério da Saúde lançou em 2011 o Plano de Ações Estratégicas para o Enfrentamento das DCNT no Brasil, 2011-2022, com o objetivo de promover o desenvolvimento e a implementação de políticas públicas efetivas, integradas, sustentáveis e baseadas em evidências para a prevenção e o controle das DCNT e seus $\mathrm{FR}^{1,8}$. No âmbito global, a Organização Mundial de Saúde (OMS) aprovou, em 2013, o Plano de Ação para a Prevenção e Controle das DCNT, que incluiu opções de políticas que implementadas coletivamente contribuem para o progresso das metas globais a serem alcançadas até $2025^{\circ}$, constituindo-se uma importante agenda multilateral para o enfrentamento das DCNT. Assim, as metas do plano nacional são coincidentes com as globais e representam um progresso significativo para o enfrentamento das DCNT e seus $\mathrm{FR}^{10}$.

Para monitorar as metas propostas e assumidas globalmente, o Brasil apresenta um sistema estruturado de vigilância de DCNT, para o qual foram implantados diferentes inquéritos, tais como o Sistema de Vigilância de Fatores de Risco e Proteção para Doenças Crônicas por Inquérito Telefônico (Vigitel), a Pesquisa Nacional de Saúde (PNS) e a Pesquisa Nacional de Saúde do Escolar (PeNSE), que possibilitam acompanhar o alcance das metas, por meio de análises de séries históricas $^{11}$. As metas devem ser objeto de contínuo monitoramento pelo país, pela sociedade civil e por instituições de saúde, ensino e pesquisa, pois contribuem para a reorientação dos serviços de saúde, da atenção primária e do processo de trabalho. Além disso, auxiliam na revisão de estratégias para a prevenção, o enfrentamento e o controle das DCNT, especialmente em um cenário de crise econômica e austeridade que pode comprometer os resultados e os indicadores de saúde.

Nesse sentindo, os objetivos deste estudo consistem em monitorar as tendências e as projeções das metas de fatores de risco e proteção para o enfrentamento das DCNT nas capitais brasileiras e também verificar se a crise econômica e as políticas de austeridade interferiram no comportamento dessas metas.

\section{Método}

Trata-se de estudo de série temporal da prevalência de FR e proteção para as DCNT. Foram utilizados os dados do Vigitel entre os anos de 2006 e 2019.

O Vigitel é um inquérito telefônico de base populacional, que monitora anualmente a frequência e a distribuição dos principais FR para DCNT. Os procedimentos de amostragem empregados visam obter a representatividade das capitais dos 26 estados brasileiros e do Distrito Federal, através de amostras probabilísticas da população de adultos ( $\geq 18$ anos de idade) que residem em domicílios com pelo menos uma linha telefônica fixa. São realizadas aproximadamente 2 mil entrevistas em cada cidade, totalizando cerca de 54 mil por ano. Em função da baixa cobertura de linhas telefônicas, são utilizados fatores de ponderação para aumentar a representatividade da população adulta de cada cidade ${ }^{12}$. Detalhes sobre o processo de amostragem e de coleta de dados são fornecidos em publicações do Vigitel ${ }^{12,13}$.

Para o presente estudo, foram utilizados os seguintes indicadores avaliados no Vigitel ${ }^{12}$, e as respectivas metas propostas no Plano de Ações Estratégicas para o Enfrentamento das DCNT 
no Brasil 2011- 2022 , e no Plano de Ação Global para a Prevenção e Controle das DCNT da OMS

Percentual de fumantes: relação de indivíduos fumantes pelo total de entrevistados. Foi considerado fumante o indivíduo que respondeu positivamente à questão: "Atualmente, o(a) Sr.(a) fuma?", independentemente do número de cigarros, da frequência e da duração do hábito de fumar. Meta: reduzir as prevalências do tabagismo em 30\%.

Percentual de adultos com obesidade: relação de indivíduos com índice de massa corporal (IMC) $\geq 30 \mathrm{~kg} / \mathrm{m}^{2}$ pelo total de entrevistados. Considerou-se obeso o indivíduo com IMC $\geq 30$ $\mathrm{kg} / \mathrm{m}^{2}$, cujas altura e peso foram autorreferidas. Meta: deter o crescimento da prevalência da obesidade.

Percentual de adultos que consomem frutas $e$ hortaliças conforme recomendado (indicador acrescentado ao Vigitel em 2008): relação de indivíduos com consumo recomendado de frutas e de hortaliças pelo total de entrevistados. Considerou-se o consumo de uma fruta ou de um suco de fruta como equivalente a uma porção, limitando-se em três o número máximo de porções diárias para frutas e em um para sucos. No caso de hortaliças, o número máximo foi de quatro porções diárias, que incluiu indivíduos que informaram o hábito de consumir saladas de hortaliças cruas, verduras e legumes cozidos no almoço e no jantar. A recomendação para o consumo de frutas e hortaliças foi considerada alcançada quando o indivíduo referiu o consumo desses alimentos em pelo menos cinco dias da semana e quando a soma das porções consumidas diariamente totalizava pelo menos cinco. Meta: aumentar o consumo de frutas e hortaliças em $10 \%$.

Percentual de adultos que praticam atividades físicas no tempo livre (AFTL) suficientemente (indicador acrescentado no Vigitel em 2009): relação de indivíduos que praticam pelo menos $150 \mathrm{mi}$ nutos semanais de atividade física de intensidade moderada ou pelo menos 75 minutos semanais de atividade física de intensidade vigorosa, pelo número de indivíduos entrevistados. Meta: aumentar a prática de atividade física em 10\%.

Percentual de adultos que consumiram bebidas alcoólicas de forma abusiva: relação de indivíduos que consumiram bebida alcoólica de forma abusiva, pelo número de indivíduos entrevistados. Foi considerado consumo abusivo de bebidas alcoólicas cinco ou mais doses (homem) ou quatro ou mais doses (mulher) em uma única ocasião, pelo menos uma vez nos últimos 30 dias. Uma dose de bebida alcoólica corresponde a uma lata de cerveja, uma taça de vinho ou uma dose de cachaça, whisky ou qualquer outra bebida alcoólica destilada. Meta: reduzir as prevalências de consumo abusivo de álcool em pelo menos $10 \%$.

Justifica-se a escolha desses indicadores por serem os FR (tabagismo, álcool e obesidade) e de proteção (consumo de frutas e hortaliças e prática de atividade física) comuns para os quatro principais grupos de DCNT (circulatórias, câncer, respiratórias crônicas e diabetes), em que a abordagem integrada desses fatores atuará nesses grupos de doenças e trará benefícios às demais $\mathrm{DCNT}^{1}$. Além disso, são contínuos no questionário do Vigitel, o que permitiu as análises de séries temporais.

Estratificaram-se os indicadores segundo sexo (feminino e masculino) e anos de escolaridade ( 0 a $8 ; 9$ a 11; e 12 anos e mais de estudo). As séries temporais foram referentes ao período de 2006 a 2019, exceto para os indicadores consumo recomendado de frutas e hortaliças e prática de AFTL, que foram incluídos em 2008 e 2009, respectivamente, em função de mudanças no questionário utilizado pelo Vigitel.

Este estudo utilizou a Série Temporal Interrompida, metodologia que tem como objetivo verificar se houve efeito de uma intervenção nas tendências temporais de uma medida de interesse analisada ${ }^{14}$. Considerando a implantação do Plano Nacional para o enfrentamento das DCNT, 2011-2022 ${ }^{1}$, a ocorrência da crise econômica com implantação de políticas de austeridade que resultaram em mudanças de indicadores ${ }^{15,16}$, foram calculadas as tendências temporais das prevalências dos FR e proteção para as DCNT de 2006 a 2019 (período completo), 2006 a 2014 (implantação do Plano e estabilidade econômica) e 2015 a 2019 (crise econômica e austeridade).

Para a análise das séries temporais, empregou-se a regressão linear generalizada de Prais -Winsten, que corrige o efeito da autocorrelação serial de primeira ordem (AR1). Considerou-se a existência de tendência significativa quando o coeficiente angular da regressão $(\beta)$, do modelo, foi diferente de zero e o valor-p inferior ou igual a 0,05 . Dessa forma, verificou-se aumento na variação percentual média anual do indicador no período, quando o foi positivo, redução se negativo e estacionária quando não identificada diferença estatisticamente significativa. Utilizouse o valor de $\mathrm{R}^{2}$ ajustado como medida de adequação para o ajuste do modelo. Para verificar a existência de autocorrelação da série, aplicou-se o teste de Durbin-Watson para todo o período (2006 a 2019). 
Foram elaboradas projeções lineares $(y=\alpha$ $+\beta \mathrm{x}$ ) de cada indicador, a partir de 2011 (implantação do plano nacional ${ }^{1}$ ) até 2025 (ano final para o cumprimento das metas globais do Plano de DCNT da $\mathrm{OMS}^{15}$ ), em um cenário no qual as metas seriam alcançadas. Para isso, consideraram-se as seguintes metas do Plano de Ações Estratégicas para o Enfrentamento das DCNT no Brasil 2011-2022': reduzir as prevalências do tabagismo em $30 \%$; deter o crescimento da prevalência da obesidade; aumentar o consumo de frutas e hortaliças em $10 \%$; aumentar a prática de atividade física em $10 \%$; e reduzir as prevalências de consumo abusivo de álcool em pelo menos $10 \%$. Para todas as metas foram feitas projeções lineares com os dados obtidos dos distintos períodos de tempo: 2006 a 2019, 2006 a 2014, e 2015 a 2019. Utilizando esses períodos de anos, também foram realizadas projeções lineares para estimar as prevalências dos indicadores até 2025.

Para análise dos dados, foram considerados os pesos pós-estratificação usados no Vigitel ${ }^{12,13}$. As análises foram realizadas no software Stata (Stata Corp LP, College Station, Texas, United States), versão 13.0. A organização dos dados e a elaboração dos gráficos foram feitas no Microsoft ${ }^{\circledR}$ Office Excel 2016.

Os dados do Vigitel estão disponíveis para acesso e uso público e sua coleta foi aprovada pela Comissão Nacional de Ética em Pesquisa para Seres Humanos do Ministério da Saúde. Obteve-se o consentimento livre e esclarecido oralmente no momento do contato telefônico com os entrevistados.

\section{Resultados}

A Figura 1 representa a série temporal das prevalências dos FR e proteção para as DCNT, segundo sexo, com a representação da interrupção, o que possibilita visualizar variações nas tendências das prevalências dos indicadores a partir de 2015. Ao considerar todo período, a tendência foi de declínio significativo $(p<0,05)$ das prevalências de fumantes (A). Em relação à obesidade (B), ao consumo recomendado de frutas e hortaliças (C) e à prática de AFTL (D), houve aumento significativo das prevalências $(p<0,05)$. Para o consumo abusivo de bebidas alcoólicas (E) a tendência foi de aumento entre as mulheres, contudo, entre homens e população total, as tendências foram de estabilidade (Figura 1).

As Tabelas 1 e 2 mostram os resultados das séries temporais interrompidas, realizadas em dois períodos (2006 a 2014 e 2015 a 2019), segundo sexo e escolaridade, respectivamente. Entre 2006 e 2014, a prevalência de fumantes reduziu de forma significativa $(\mathrm{p}<0,05)$ na população total $(\beta$ $=-0,64)$, entre homens $(\beta=-0,79)$ e mulheres $(\beta=-0,50)$, bem como em todos os níveis de escolaridade. No segundo período (2015 a 2019), as tendências continuaram declinando, contudo, com menor taxa de redução ao se comparar com o primeiro período. A obesidade teve crescimento significativo $(p<0,05)$ nos dois períodos analisados, porém a taxa de crescimento foi menor entre 2015 e 2019. O consumo de frutas e hortaliças aumentou entre 2006 e 2014 para ambos os sexos e para o total da população, bem como para todos os níveis de escolaridade $(\mathrm{p}<0,05)$. Em contrapartida, no segundo período inverteram-se as tendências, com redução significativa desse consumo $(\mathrm{p}<0,05)$ para população total $(\beta=-0,59)$, sexo masculino $(\beta=-0,62)$, sexo feminino $(\beta=-0,58)$, indivíduos com 0 a 8 anos de estudo $(\beta=-0,25)$ e 9 a 11 anos $(\beta=-0,83)$, mantendo-se estável entre os mais escolarizados $(\beta=-1,16 ; p=0,08)$. A prática de AFTL teve aumento significativo no primeiro período para ambos os sexos e para todos os anos de estudo (p $<0,05)$. Entretanto, no segundo período, a tendência foi de estabilidade para a populaçãototal $(\mathrm{p}=0,20)$, com menor taxa de crescimento entre os homens $(\beta=0,03)$ e mulheres $(\beta=0,59) \mathrm{em}$ relação ao primeiro período analisado. Ocorreu estabilidade, também, das prevalências da prática de AFTL para todos os níveis de estudo ( $p>0,05)$, com inversão da inclinação entre aqueles com 9 a 11 anos $(\beta=-0,08 ; p=0,06)$. Quanto ao consumo abusivo de bebidas alcoólicas, de 2006 a 2014, as tendências foram estáveis para o total da população, para ambos os sexos, e 0 a 8 anos de estudo $(p>0,05)$. Houve uma pequena redução das prevalências desse consumo para aqueles com 9 a 11 anos de escolaridade $(\beta=-0,01 ; \mathrm{p}=0,04)$ e aumento entre indivíduos com 12 anos ou mais $(\beta=1,35 ; p<0,01)$. Entre 2015 e 2019 , alterouse o cenário, havendo aumento significativo do consumo de bebidas alcóolicas entre as mulheres $(\beta=0,32)$, na população total $(\beta=0,13)$ e nos indivíduos com 9 a 11 anos de estudo $(\beta=0,33)$; por outro lado, ocorreu redução $(\mathrm{p}<0,05)$ entre os homens $(\beta=-0,15)$, com escolaridade de 0 a 8 anos $(\beta=-0,29)$ e 12 anos ou mais $(\beta=-0,06)$ (Tabelas 1 e 2).

A Figura 2 apresenta as projeções das metas dos FR e a proteção para as DCNT até o ano de 2025. A redução da prevalência de tabagismo em $30 \%$ (A) poderá ser atingida em todos cenários 
(A)

(B)
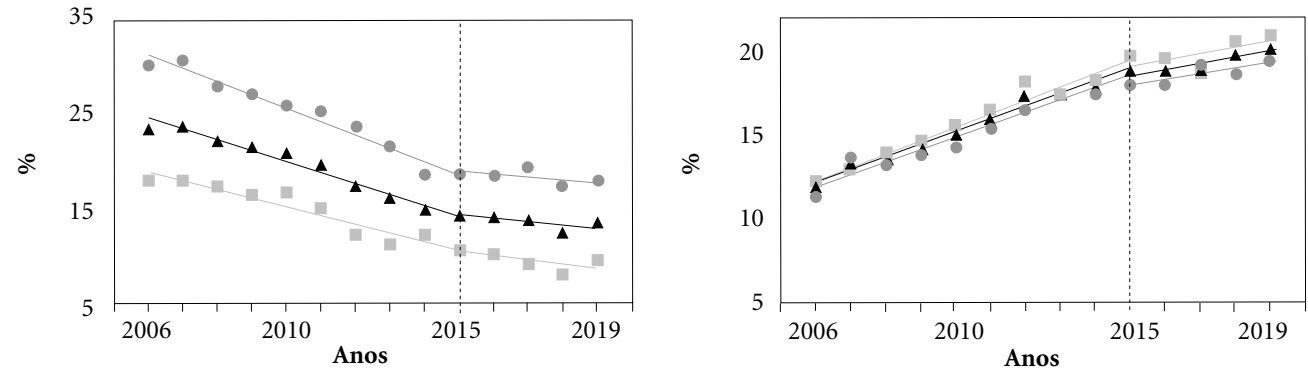

(C)

(D)
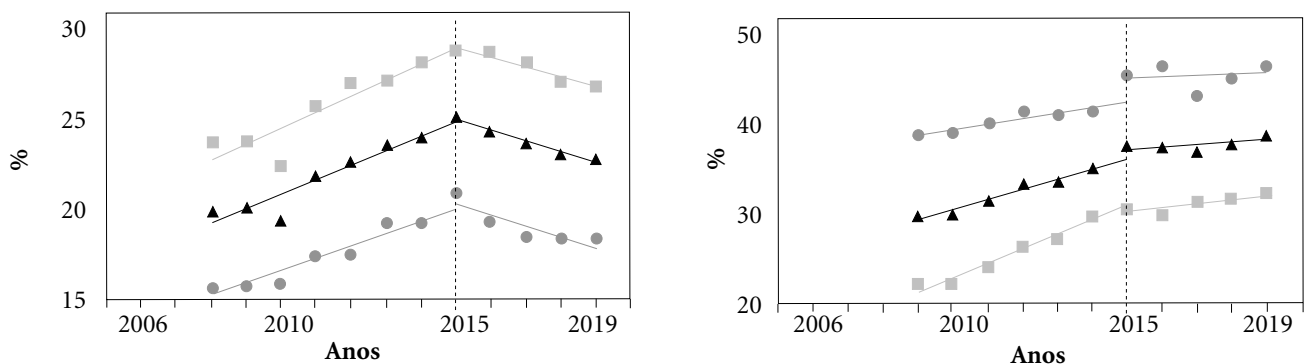

(E)

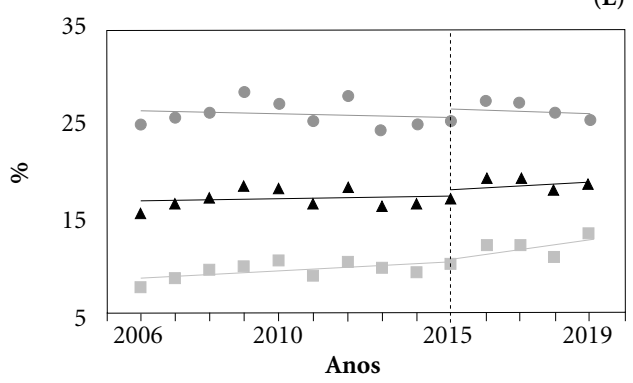

- Masculino

- Feminino

\ Total

Figura 1. Tendência temporal das prevalências de tabagismo, obesidade, consumo recomendado de frutas e hortaliças, prática de atividade física no tempo livre e consumo abusivo de bebidas alcoólicas. Vigitel. Capitais brasileiras, 2006 a 2019.

(A) = Prevalência de tabagismo; $(\mathrm{B})$ = Prevalência de obesidade; $(\mathrm{C})=$ Prevalência do consumo recomendado de frutas e hortaliças; (D) = Prevalência da prática de atividade física no tempo livre; (E) = Prevalência do consumo abusivo de bebidas alcoólicas.

avaliados. A meta de deter o crescimento da obesidade (B) não seria cumprida em nenhum cenário. $\mathrm{O}$ aumento do consumo de frutas e hortaliças em 10\% (C) poderá não ser alcançada, se considerado o período de 2015 a 2019. Aumentar a prática de AFTL em 10\% (D) também seria atingida em todos os cenários analisados. A redução do consumo abusivo de bebidas alcoólicas em pelo menos 10\% (E) não seria possível em nenhum cenário (Figura 2).

\section{Discussão}

De 2006 a 2019, nas capitais brasileiras, ocorreu redução da prevalência de fumantes, aumento da obesidade, do consumo de frutas e hortaliças e da prática de AFTL, bem como estabilidade do consumo abusivo de bebidas alcoólicas. No geral, ao analisar pelas séries temporais interrompidas, entre 2015 e 2019, os indicadores demonstraram pior desempenho na comparação com os anos 
anteriores: menor taxa de redução das prevalências de fumantes, declínio do consumo de frutas e hortaliças, estabilidade da prática de AFTL e aumento do consumo abusivo de álcool. As projeções para 2025 evidenciaram que as metas de deter o crescimento da obesidade e de reduzir o uso abusivo de bebidas alcoólicas não seriam atingidas, enquanto o aumento do consumo de frutas e hortaliças não será alcançada se considerar o período de 2015 a 2019. Para os demais indicadores, as metas deverão ser cumpridas.

A melhoria dos indicadores entre 2006 e 2014 está relacionada com o contexto político e econômico favorável, que permitiu investimentos na saúde e maior financiamento público para o Sistema Único de Saúde (SUS), contribuindo para o fortalecimento da sua capacidade de resposta ao enfrentamento das DCNT. O Ministério da Saúde instituiu diversas políticas, programas, ações e estratégias, com destaque para a organização da Vigilância das DCNT e dos $\mathrm{FR}^{8}$ e a implementação dos inquéritos populacionais (domiciliares, telefônicos e escolares) ${ }^{11}$. Adicionalmente, a Política Nacional de Promoção da Saúde (PNPS) estabelece como ações prioritárias a alimentação adequada e saudável, as práticas corporais e as atividades físicas, bem como a prevenção de FR à saúde ${ }^{17}$. Ocorreu também a expansão da Atenção Primária em Saúde (APS) e da Atenção Farmacêutica, com o Programa Farmácia Popular ${ }^{8}$. Todas essas medidas estão inseridas no contexto da implementação do Plano de Ações Estratégicas para o Enfrentamento das DCNT, 2011-2022, e alinhadas com o plano global e com a Agenda $2030^{1,8,9}$.

O estudo mostrou piora de alguns indicadores a partir de 2015, o que pode ser consequência da crise econômica e da austeridade no Brasil, as quais impactaram na redução do produto interno bruto (PIB), no aumento do desemprego e das desigualdades, além de afetar diretamente os serviços de saúde. Houve redução do PIB em 2015 $(-3,5 \%)$ e $2016(-3,3 \%)$, com uma lenta recuperação nos anos seguintes: 2017 (+1,3\%), 2018 $(+1,3 \%)$ e $2019(+1,1 \%)^{18}$. Entre 2014 e 2019 , a população desocupada passou de 6,7 para 12,6 milhões e, em 2017, foi registrada a maior taxa de desocupação $(12,7 \%)^{19,20}$. O Índice de Gini do rendimento médio mensal domiciliar per capita entre 2012 e 2015 reduziu-se de 0,540 para 0,524; em 2016, aumentou para 0,537, chegando a 0,545 em 2018 e 0,543 em $2019^{21}$. Soma-se ainda as políticas de austeridade que foram implantadas, como a aprovação da Emenda Constitucional 95, piorou o quadro, com redução de investimentos 
em políticas sociais e de saúde, na ciência, na tecnologia e na regulação de produtos ${ }^{22,23}$. Sabe-se que as decisões políticas em como responder à crise econômica têm efeitos pronunciados e não intencionais na Saúde Publica ${ }^{24}$, pois o aumento do desemprego e da inflação, além do ajuste fiscal e da austeridade, estão associados a deteriorações duradouras em vários indicadores de saúde da população, como aumento da mortalidade em adultos $^{25}$ e da morbimortalidade infantil ${ }^{26}$, além dos impactos negativos para o alcance das metas dos Objetivos do Desenvolvimento Sustentável $(\mathrm{ODS})^{27}$.

Houve diminuição da prevalência de fumantes, porém, no entanto, entre 2015 e 2019, a taxa de redução foi menor na comparação com os anos anteriores. Globalmente, a prevalência de tabagismo em 2015 foi de 25,0\% entre os homens e $5,4 \%$ entre as mulheres, e $11,5 \%$ das mortes (6,4 milhões) foram atribuídas ao tabagismo ${ }^{28}$. No Brasil, de acordo com a PNS, a prevalência de tabagismo na população adulta foi de $14,7 \%{ }^{29}$. Por conseguinte, diversas medidas regulatórias selaram o compromisso do Brasil para sua redução e, com isso, houve diminuição da prevalência de fumantes no país ${ }^{30}$. Evidenciam-se a ratificação da Convenção-Quadro para o Controle do Tabaco, os ambientes livres de tabaco, a definição de preço mínimo para comercialização, a proibição da promoção, do patrocínio, da venda a menor de 18 anos e da propaganda comercial de produto fumígeno, a atualização das diretrizes de cuidado à pessoa tabagista no âmbito do SUS, entre outras ${ }^{30}$. No entanto, há um enfraquecimento do papel regulatório do governo e a necessidade da implementação de novas medidas, da fiscalização dos ambientes, dos pontos de vendas e do comércio ilegal, da vigilância das atividades e políticas ${ }^{22}$. Estudos realizados na Itália $^{31}$ e nos Estados Unidos ${ }^{32}$ também observaram aumento da prevalência de tabagismo em decorrência da crise econômica, sendo justificado pelo suposto efeito de reduzir o estresse, causado por problemas financeiros e de desemprego ${ }^{31,32}$. Em contrapartida, outro estudo, realizado na Itália, entre 2016 e 2017, mostrou redução do hábito de fumar em decorrência do menor poder de com$\mathrm{pra}^{33}$. Assim, um período de crise pode aumentar o tabagismo ou impedir que algumas pessoas comecem a fumar ou fazer com que outras abandonem o hábito ${ }^{33}$.

A obesidade aumentou em todos os períodos analisados e, de acordo com as projeções, a meta de deter o seu crescimento não seria alcançada até 2025. O aumento da obesidade mostra pa-

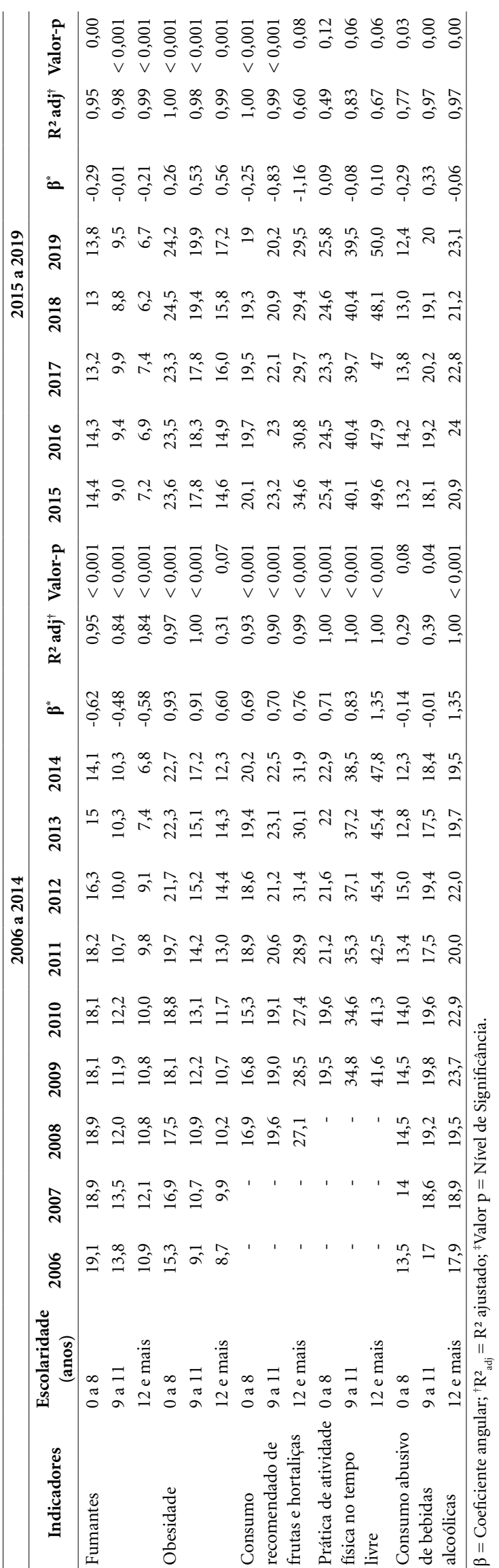


(A)

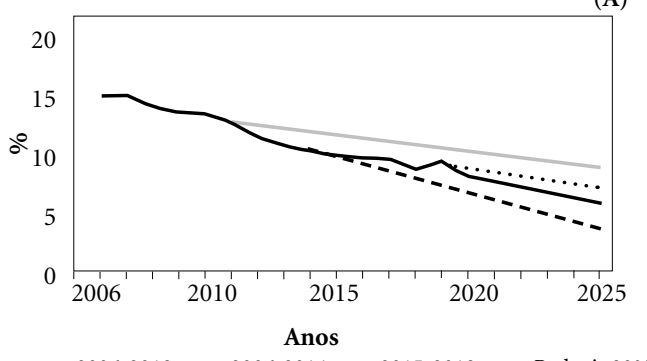

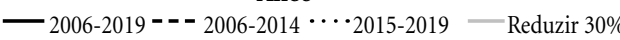

(C)

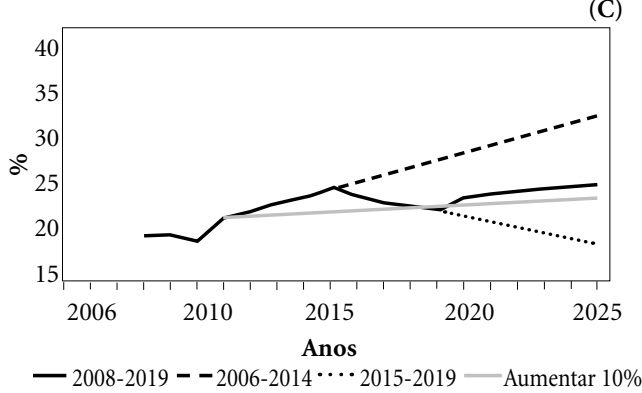

(B)

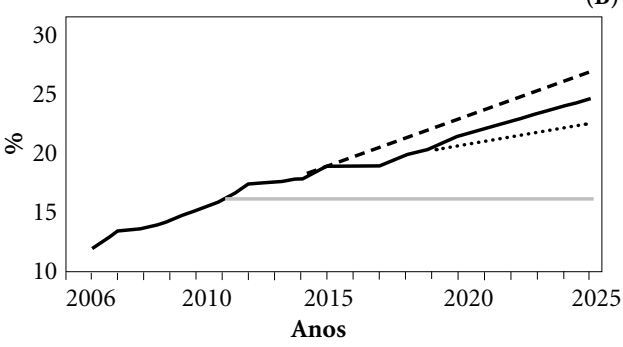

— 2006-2019- -2006-2014ㄴ.2015-2019 — Deter o crescimento

(D)

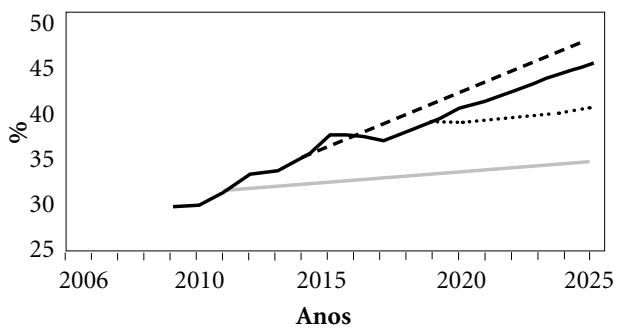

2009-2019 ---2006-2014 …2015-2019 —Aumentar 10\%

(E)

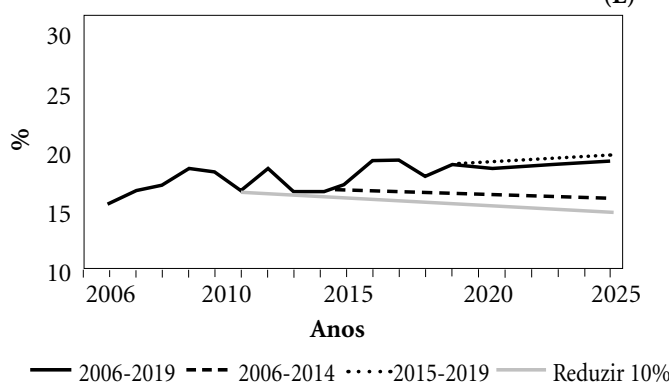

Figura 2. Projeções das prevalências de tabagismo, obesidade, consumo recomendado de frutas e hortaliças, prática de atividade física no tempo livre e consumo abusivo de bebidas alcoólicas. Vigitel. Capitais brasileiras, 2006 a 2025.

(A) = Tabagismo; $(\mathrm{B})$ = Obesidade; $(\mathrm{C})$ = Consumo recomendado de frutas e hortaliças; (D) = Prática de atividade física no tempo livre; $(\mathrm{E})=$ Consumo abusivo de bebidas alcoólicas.

drões inadequados de alimentação e de atividade física. Em 2016, havia aproximadamente 650 milhões de obesos no mundo ${ }^{34}$, enquanto que no Brasil, em 2013, as prevalências de obesidade foram de $16,8 \%$ para homens e $24,4 \%$ para mulheres $^{35}$. A população brasileira, nas últimas décadas, passou por transformações sociais e econômicas que resultaram em mudanças no seu padrão de saúde e de consumo alimentar e, por isso, houve aumento do excesso de peso e da obesidade em todas as camadas da população, apontando para um novo cenário de problemas relacionados à alimentação e nutrição ${ }^{36}$. As causas da obesidade são multifatoriais, incluindo fatores comportamentais, ambientais, socioeconômicos e genéti$\cos ^{37}$. Tem-se ainda a disseminação do fast food, dos ambientes obesogênicos e do aumento do consumo de alimentos ultraprocessados, uma vez que esses tendem a crescer em épocas de crise por terem um menor preço, ao se comparar com o dos alimentos frescos ${ }^{38,39}$.

As frutas e as hortaliças são indispensáveis para um padrão saudável de alimentação. No entanto, o baixo consumo é considerado um dos 
principais fatores de risco dietético para a mortalidade e aos anos de vida ajustados por incapacidade (disability adjusted life of years - DALYs) ${ }^{40}$. Em 2017, cerca de 2 milhões de óbitos no mundo foram atribuídos ao baixo consumo desses alimentos ${ }^{40}$. No Brasil, de acordo com a PNS, apenas $37,3 \%$ da população adulta consumiam frutas e hortaliças em cinco ou mais vezes ao $\mathrm{dia}^{41}$. A evolução positiva da prevalência do consumo de frutas e hortaliças no primeiro período analisado neste estudo (2006 a 2014) deve-se, em parte, a um cenário mais favorável para a promoção da alimentação saudável no Brasil, pelas ações do Governo Federal para a segurança alimentar e nutricional, tais como: o Programa de Aquisição de Alimentos, o Plano Nacional de Segurança Alimentar e Nutricional, a Política Nacional de Alimentação e Nutrição, o Guia Alimentar para a População Brasileira e os livros Alimentos Regionais Brasileiros e $\mathrm{Na}$ Cozinha com as Frutas, Legumes e Verduras ${ }^{42}$. Somam-se, ainda, os fatores econômicos como a renda familiar e o melhor preço desses alimentos, visto que, nesse período, houve crescimento econômico no Brasil ${ }^{12,43}$. Um estudo observou o aumento da participação de frutas e hortaliças no total de aquisições de alimentos, quando houve a diminuição do preço ou o aumento da renda familiar ${ }^{43}$. Esses fatores econômicos explicariam também o decréscimo do consumo entre 2015 e 2019, visto que houve elevação nos preços dos alimentos, diminuição da renda e aumento do desemprego em razão da crise econômica no país ${ }^{42}$. Sabe-se que a renda dos brasileiros e o preço dos alimentos são fatores que afetam diretamente a acessibilidade alimentar e são determinantes para o consumo de alimentos saudáveis ${ }^{44}$.

No contexto de aumento da obesidade e da redução do consumo de frutas e hortaliças, tornam-se necessários maiores avanços e investimentos em medidas regulatórias para a taxação das bebidas açucaradas, subsídios aos alimentos saudáveis, eliminação das gorduras trans da produção industrial e proibição do marketing de alimentos para crianças, sendo essas medidas preconizadas pela OMS para reduzir a dieta não saudáve ${ }^{45}$. Ademais, estratégias de regulamentação do mercado que oportunizem a produção e a distribuição de frutas e hortaliças, como o Programa de Aquisição de Alimentos e a inserção de feiras-livres, poderiam solucionar questões como a disparidade do acesso físico e financeiro a esses alimentos $^{42,43}$. Tem-se, ainda, a necessidade de se estabelecer um conjunto de políticas que agregue todos os conteúdos que determinam a segurança alimentar no Brasil, além de políticas macroeconômicas coerentes com a geração de emprego e renda, e que sejam sinérgicas às políticas sociais, para que se possa caminhar no sentido da superação da pobreza ${ }^{44}$.

As evidências sobre os benefícios da atividade física para a saúde estão estabelecidas desde $1950^{46}$. Todavia, em 2016, 27,5\% da população adulta no mundo era insuficientemente ativa, ou seja, não atingiam as recomendações de pelo menos 150 minutos semanais de $\mathrm{AFTL}^{47}$. No Brasil, em $2013,46,0 \%$ da população adulta era insuficientemente ativa e apenas $22,5 \%$ atingiram as recomendações de $\mathrm{AFTL}^{48}$. A prática de AFTL elevou-se de 2006 a 2014 e, após, houve estabilidade para população total, tornando-se um cenário preocupante, visto que a inatividade física é um dos principais fatores de risco para DCNT, pois reduz a expectativa de vida, e afeta negativamente a saúde mental e a qualidade de vida ${ }^{47}$. Para contribuir com a promoção da saúde da população, em 2011, foi implantado o Programa Academia da Saúde (PAS), no âmbito do SUS ${ }^{49}$. O PAS ampliou o acesso e oportunizou a prática de atividade física para todos, inclusive para os mais vulneráveis ${ }^{50}$. Contudo, o PAS tem apresentado desafios no cenário atual, como dificuldades para a ampliação do número de polos em todo país, a manutenção das atividades e a sustentabilidade do programa nas redes de serviços dos municípios $^{49}$. As crises econômicas podem estar associadas a mudanças no estilo de vida, acarretando em redução da prática de atividade física ${ }^{44}$. Por outro lado, estudos apontam que a média de horas trabalhadas pode diminuir durante as crises econômicas, aumentando o tempo disponível para o envolvimento em atividades de promoção da saúde, incluindo exercício e atividade física ${ }^{51,52}$.

O consumo abusivo de bebidas alcoólicas manteve-se estável entre 2006 e 2014, contudo, o cenário se alterou a partir de 2015 , com aumento do uso na população total e entre as mulheres. $\mathrm{O}$ álcool é uma causa importante de morte prematura no Brasil e, além de ser um FR para diversas doenças e agravos em saúde ${ }^{53}$ foi responsável por $10 \%$ de todas as mortes no mundo entre indivíduos de 15 a 49 anos de idade ${ }^{54}$. De acordo com dados da PNS, a prevalência do consumo abusivo de bebidas alcoólicas no Brasil foi de 13,7\% $\%^{55}$. Globalmente é observado um aumento no consumo abusivo de álcool, com uma tendência de crescimento entre o sexo feminino ${ }^{56}$. A hipótese para esse fato está relacionada ao crescimento da autonomia das mulheres, pela maior participação no mercado de trabalho e educação, o que 
possibilita e encoraja a consumirem bebidas alcoólicas ${ }^{56}$. Esse consumo também tende a ser maior entre aqueles com maior nível socioeconômico, contudo, os efeitos adversos do uso abusivo de álcool são maiores entre os indivíduos com menor nível socioeconômico ${ }^{56}$. Há, ainda, as questões relacionadas com as mudanças desse consumo em períodos de $\mathrm{crises}^{57}$. Uma revisão sistemática evidenciou dois mecanismos comportamentais pelos quais as crises econômicas podem influenciar o uso do álcool. O primeiro mecanismo indica que o sofrimento psicológico desencadeado pelo desemprego e pela redução de renda pode aumentar alcoolismo; já o segundo sugere que restrições orçamentárias acarretam em menor gasto com bebidas alcoólicas ${ }^{58}$. No Brasil, diversas iniciativas foram adotadas para coibir o consumo abusivo de álcool: o fortalecimento da implementação da política de preços e de aumento de impostos, apoio à intensificação de ações fiscalizatórias em relação à venda de bebidas alcoólicas a menores de 18 anos, bem como das ações educativas voltadas à prevenção e à redução do uso de álcool, e medidas de monitoramento e vigilância ${ }^{8}$. Torna-se, porém, necessário avançar em novas medidas regulatórias, proibições ou restrições mais abrangentes às propagandas de álcool na mídia, redução de horários e pontos de venda e mudanças na Lei No 9.294/1996, que considera bebidas alcoólicas aquelas com teor alcoólico superior a $13^{\circ}$ Gay Lussac para englobar as cervejas e outras bebidas ${ }^{22,59}$.

Outra questão preocupante está relacionada aos determinantes sociais, como o nível educacional, que agrava a carga de doenças em populações vulneráveis ${ }^{60}$. As DCNT e os FR se distribuem desigualmente entre os estratos de escolaridade. Nesse sentido, indivíduos com menor nível de escolaridade apresentam maiores prevalências de DCNT, de FR e de incapacidades causadas por essas doenças ${ }^{60-62}$. O baixo nível de escolaridade pode limitar o acesso às informações e aos hábitos de vida mais saudáveis, dificultar a compreensão sobre a gravidade da doença e a adesão ao tratamento ${ }^{63,64}$.

As crises econômicas e a austeridade podem aumentar o uso dos serviços, as desigualdades de saúde ${ }^{15}$ e afetar os padrões comportamentais e os estilos de vida da população $0^{31,51,52,58}$. O cenário atual da pandemia do Coronavírus Disease 2019 (Covid-19) também está acarretando mudanças nos indicadores de saúde e, consequentemente, na piora nos padrões de saúde da população, com aumento dos FR e redução dos fatores de proteção para as DCNT, o que é ainda mais grave em países com situação de extrema vulnerabilidade, com altas taxas de desemprego e que tiveram cortes nas políticas sociais ${ }^{65}$. Esse contexto exige a integração de políticas públicas intersetoriais, o avanço para a redução de desigualdades, o fortalecimento das medidas de proteção e de promoção da saúde e o monitoramento contínuo das DCNT, seus FR, e das metas assumidas pelos planos nacional e global.

Este estudo tem como limitações os dados serem coletados de forma autorreferida, que podem resultar na sub ou superestimação das prevalências reais e gerar estimativas menos precisas. Porém, estudos de validação do questionário do Vigitel mostram resultados satisfatórios nas análises reprodutibilidade e validade ${ }^{66-68}$. O fato da amostra do Vigitel ser composta apenas por indivíduos residentes nas capitais dos estados brasileiros e no Distrito Federal, que residem em domicílios com telefone fixo, representa um risco potencial à representatividade da amostra. Porém, essa questão é minimizada pelo uso de fatores de ponderação dos dados, os quais buscam igualar as características demográficas da amostra do Vigitel às características da população total, segundo os dados do censo do Instituto Brasileiro de Geografia e Estatística (IBGE) ${ }^{12,13}$. Além disso, a análise de série interrompida de 2015 a 2019 ainda é limitada, por isso, são necessárias observações futuras para verificar a tendência desses indicadores.

\section{Conclusão}

As análises de séries temporais interrompidas possibilitaram evidenciar mudanças nas tendências dos fatores de risco e de proteção para as DCNT, ao considerar os dois períodos de tempo: 2006 a 2014 (implantação do plano de DCNT e estabilidade econômica) e 2015 a 2019 (crise econômica e austeridade). No primeiro período, houve redução da prevalência de fumantes, aumento da obesidade, do consumo de frutas e hortaliças e da prática de AFTL, bem como estabilidade do consumo abusivo de bebidas alcoólicas. No entanto, após 2015, observou-se piora nos indicadores ao se comparar com os anos anteriores, com menor taxa de redução das prevalências de fumantes, declínio do consumo de frutas e hortaliças, estabilidade da prática de AFTL e aumento do consumo abusivo de álcool. De acordo com as projeções, as metas de deter a obesidade e reduzir o uso de álcool não seriam atingidas. Por isso, torna-se importante reforçar 
as ações de promoção e de incentivo a hábitos de vida saudável, avançar nas medidas regulatórias e na sustentabilidade das ações, programas e políticas para o enfrentamento das DCNT no Brasil.
O monitoramento desses indicadores deve ser contínuo, especialmente em tempos de instabilidade político-econômica e de ameaça a direitos sociais e da saúde.

\section{Colaboradores}

AG Silva, RA Teixeira e DC Malta trabalharam na concepção, o delineamento, análise e interpretação dos dados, na redação e revisão crítica do artigo e na aprovação da versão a ser publicada. EJS Prates trabalhou na interpretação dos dados, na redação e revisão crítica do artigo e na aprovação da versão a ser publicada.

\section{Agradecimentos}

À Coordenação de Aperfeiçoamento de Pessoal de Nível Superior (CAPES), pela bolsa de doutorado para Alanna Gomes Silva. Ao Conselho Nacional de Desenvolvimento Científico e Tecnológico $(\mathrm{CNPq})$, pela bolsa de produtividade para Deborah Carvalho Malta. Ao Fundo Nacional de Saúde do Ministério da Saúde pela bolsa de pesquisa para Elton Junio Sady Prates. 


\section{Referências}

1. Brasil. Ministério da Saúde (MS). Plano de ações estratégicas para o enfrentamento das doenças crônicas não transmissíveis (DCNT) no Brasil 2011-2022. Brasília: MS; 2011.

2. World Health Organization (WHO). World health statistics 2018: monitoring health for the SDGs, sustainable development goals. Geneva: WHO; 2019. [cited 2020 Jan 19]:1-86. Available from: https:// apps.who.int/iris/bitstream/handle/10665/272596/ 9789241565585-eng.pdf?ua=1

3. Pan American Health Organization (PAHO). Noncommunicable diseases in the region of the americas: facts and figures [Internet] 2019 [cited 2020 Jan 19]. Available from: http://iris.paho.org/xmlui/bitstream/ handle/123456789/51483/PAHONMH19016_eng. pdf?sequence $=6$ \&isAllowed $=y$

4. Hatefi A, Allen LN, Bollyky TJ, Roache SA, Nugent R. Global susceptibility and response to noncommunicable diseases. Bull World Health Organ 2018; 96(8):586-588.

5. Stringhini S, Carmeli C, Jokela M, Avendaño $M$, Muennig P, Guida F, Ricceri F, d'Errico A, Barros H, Bochud M, Chadeau-Hyam M, Clavel-Chapelon F, Costa G, Delpierre C, Fraga S, Goldberg M, Giles GG, Krogh V, Kelly-Irving M, Layte R, Lasserre AM, Marmot MG, Preisig M, Shipley MJ, Vollenweider P, Zins M, Kawachi I, Steptoe A, Mackenbach JP, Vineis P, Kivimäki M. Socioeconomic status and the $25 \times 25$ risk factors as determinants of premature mortality: a multicohort study and meta-analysis of 1.7 million men and women. Lancet 2017; 389(10075):1229-1237.

6. Paes-Sousa R, Schramm JMA, Mendes LVP. Fiscal austerity and the health sector: the cost of adjustments. Cien Saude Colet 2019; 24(12):4375-4384.

7. Paes-Sousa R, Rasella D, Carepa-Sousa J. Economic policy and public health: fiscal balance and population wellbeing. Saúde debate 2018; 42(n. esp. 3):172182.

8. Malta DC, Morais Neto OL, Silva Junior JB. Presentation of the strategic action plan for coping with chronic diseases in Brazil from 2011 to 2022. Epidemiol. Serv. Saúde 2011; 20(4):425-438.

9. World Health Organization (WHO). Global action plan for the prevention and control of noncommunicable diseases 2013-2020. Genebra: WHO; 2013. [cited 2020 Jan 19]. Available from: https://apps.who.int/ iris/bitstream/handle/10665/94384/9789241506236_ eng.pdf? sequence $=1$

10. Malta DC, Silva Júnior JB. Brazilian Strategic Action Plan to Combat Chronic Non-communicable Diseases and the global targets set to confront these diseases by 2025: a review. Epidemiol. Serv. Saúde 2013; 22(1):151-164.

11. Malta DC, Silva MMA, Moura L, Morais Neto OL. The implantation of the Surveillance System for Non-communicable Diseases in Brazil, 2003 to 2015: successes and challenges. Rev. bras. epidemiol 2017; 20(4):661-675.
12. Brasil. Ministério da Saúde (MS). Vigitel Brasil 2019: vigilância de fatores de risco e proteção para doenças crônicas por inquérito telefônico: estimativas sobre frequência e distribuição sociodemográfica de fatores de risco e proteção para doenças crônicas nas capitais dos 26 estados brasileiros e no Distrito Federal em 2019. Brasília: MS; 2020.

13. Bernal RTI, Iser BPM, Malta DC, Claro RM. Surveillance System for Risk and Protective Factors for Chronic Diseases by Telephone Survey (Vigitel): changes in weighting methodology. Epidemiol. Serv. Saúde 2017; 26(4):701-712.

14. Bernal JL, Cummins S, Gasparrini A. Interrupted time series regression for the evaluation of public health interventions: a tutorial. Int J Epidemiol 2017; 46(1):348-355.

15. Massuda A, Hone T, Leles FAG, Castro MC, Atun R. The Brazilian health system at crossroads: progress, crisis and resilience. BMJ Glob Health 2018; 3(4):e000829.

16. Hone T, Mirelman AJ, Rasella D, Paes-Sousa R, Barreto ML, Rocha R, Millett C. Effect of economic recession and impact of health and social protection expenditures on adult mortality: a longitudinal analysis of 5565 Brazilian municipalities. Lancet 2019; 7(11):1575-1583.

17. Malta DC, Morais Neto OL, Silva MMA, Rocha D, Castro AM, Reis AAC, Akerman M. National Health Promotion Policy (PNPS):chapters of a journey still under construction. Cien Saude Colet 2016; 21(6):1683-1694.

18. Instituto Brasileiro de Geografia e Estatística (IBGE). PIB cresce 1,1\% em 2019 e fecha o ano em $R \$ 7,3$ trilhões. Rio de Janeiro: Agência IBGE notícias; 2020.

19. Instituto Brasileiro de Geografia e Estatística (IBGE). PNAD Contínua: taxa de desocupação é de 11,0\% e taxa de subutilização é de 23,0\% no trimestre encerrado em dezembro. Rio de Janeiro: Agência IBGE notícias; 2020.

20. Instituto Brasileiro de Geografia e Estatística (IBGE). PIB cresce 1,1\% em 2018 e fecha ano em $R \$ 6,8$ trilhões. Rio de Janeiro: Agência IBGE notícias; 2019.

21. Instituto Brasileiro de Geografia e Estatística (IBGE). PNAD Contínua 2019: rendimento do 1\% que ganha mais equivale a 33,7 vezes o da metade da população que ganha menos. Rio de Janeiro: Agência IBGE notícias; 2020.

22. Malta DC, Duncan BB, Barros MBA, Katikireddi SV, Souza FM, Silva AG, Machado DB, Barreto ML. Fiscal austerity measures hamper noncommunicable disease control goals in Brazil. Cien Saude Colet 2018; 23(10):3115-3122.

23. Malta DC, Silva AG, Teixeira RA, Machado IE, Coelho MRS, Hartz ZM. Evaluation of the achievement of the goals of the Strategic Action Plan for Coping with Chronic Diseases in Brazil, 2011-2022. An Inst Hig Med Trop (Lisb) 2019; (1):9-16.

24. Karanikolos M, Mladovsky P, Cylus J, Thomson S, Basu S, Stuckler D, Mackenbach JP, McKee M. Financial Crisis, Austerity, and Health in Europe. Lancet 2013; 381(9874):1323-1331. 
25. Williams C, Gilbert BJ, Zeltner T, Watkins J, Atun R, Maruthappu M. Effects of economic crises on population health outcomes in Latin America, 1981-2010: an ecological study. BMJ Open 2016; 6(1):e007546.

26. Rasella D, Basu S, Hone T, Paes-Sousa R, Ocké-Reis $\mathrm{CO}$, Millett C. Child morbidity and mortality associated with alternative policy responses to the economic crisis in Brazil: A nationwide microsimulation study. PLoS Med 2018; 15(5):e1002570.

27. Souza LEPF, Barros RD, Barreto ML, Katikireddi SV, Hone TV, Paes de Sousa R, Leyland A, Rasella D, Millett CJ, Pescarini J. The potential impact of austerity on attainment of the Sustainable Development Goals in Brazil. BMJ Glob Health 2019; 4(5):e001661.

28. (Global Burden of Disease) GBD 2015 Tobacco Collaborators. Smoking prevalence and attributable disease burden in 195 countries and territories, 1990-2015: a systematic analysis from the Global Burden of Disease Study 2015. Lancet 2017; 389(10082):1885-1906.

29. Malta DC, Vieira ML, Szwarcwald CL, Caixeta R, Brito SMF, Reis AAC. Smoking Trends among Brazilian population - National Household Survey, 2008 and the National Health Survey, 2013. Rev. bras. epidemiol 2015; 18(Supl. 2):45-56.

30. Malta DC, Silva AG, Machado IE, Sá ACMGN, Santos FM, Prates EJS, Cristo E.B. Trends in smoking prevalence in all Brazilian capitals between 2006 and 2017. J. bras. Pneumol 2019; 45(5):e20180384.

31. Mattei G, De Vogli R, Ferrari S, Pingani L, Rigatelli M, Galeazzi GM. Impact of the economic crisis on health-related behaviors in Italy. Int J Soc Psychiatry 2017; 63(7):649-656

32. Gallus S, Ghislandi S, Muttarak R, Bosetti C. Effects of the economic crisis on smoking prevalence and number of smokers in the USA. Tob Control 2015; 24(1):82-88.

33. Petrelli F, Grappasonni I, Peroni A, Kracmarova L, Scuri S. Survey about the potential effects of economic downturn on alcohol consumption, smoking and quality of life in a sample of Central Italy population. Acta Biomed 2018; 89(1):93-98.

34. Ruban A, Stoenchev K, Ashrafian H, Teare J. Current treatments for obesity. Clin Med (Lond) 2019; 19(3):205-212.

35. Ferreira APS, Szwarcwald CL, Damacena GN. Prevalence of obesity and associated factors in the Brazilian population: a study of data from the 2013 National Health Survey. Rev. bras. Epidemiol 2019; 22:e190024.

36. Jaime PC, Delmuè DCC, Campello T, Silva DO, Santos LMP. A look at the food and nutrition agenda over thirty years of the Unified Health System. Cien Saude Colet 2018; 23(6):1829-1836.

37. Hruby A, Hu FB. The Epidemiology of Obesity: A Big Picture. Pharmacoeconomics 2015; 33(7):673-689.

38. Claro RM, Maia EG, Costa BVL, Diniz DP. Food prices in Brazil: prefer cooking to ultra-processed foods. Cad Saude Publica 2016; 32(8):e00104715.

39. Martins APB, Levy RB, Claro RM, Moubarac JC, Monteiro CA. Increased contribution of ultra-processed food products in the Brazilian diet (1987-2009). Rev Saude Publica 2013; 47(4):656-665.
40. Global Burden of Disease (GBD) 2017 Diet Collaborators. Health effects of dietary risks in 195 countries, 1990-2017: a systematic analysis for the Global Burden of Disease Study 2017. Lancet 2019; 393(10184):19581972.

41. Jaime PC, Stopa SR, Oliveira TP, Vieira ML, Szwarcwald CL, Malta DC. Prevalence and sociodemographic distribution of healthy eating markers, National Health Survey, Brazil 2013. Epidemiol. Serv. Saúde 2015; 24(2):267-276

42. Silva LES, Claro RM. Time trends in the consumption of fruits and vegetables among adults in Brazilian state capitals and the Federal District, 2008-2016. Cad Saude Publica 2019; 35(5):e00023618.

43. Claro RM, Monteiro CA. Family income, food prices, and household purchases of fruits and vegetables in Brazil. Rev Saude Publica 2010; 44(6):1014-1020.

44. Neder H, Alves FN, Souza S. Acesso à Renda e Inflação de Preços de Alimentos no Brasil: análise dos efeitos do programa Bolsa Família. Rev. Econ. Sociol. Rural 2015;53(1):51-70.

45. World Health Organization (WHO). 'Best buys' and other recommended interventions for the prevention and control of noncommunicable diseases. Genebra: WHO; 2011. [cited Mar 17 2020]. Available from: https:// apps.who.int/iris/bitstream/handle/10665/259232/ WHO-NMH-NVI-17.9-eng.pdf

46. Kohl HW, Craig CL, Lambert EV, Inoue S, Alkandari JR, Leetongin G, Kahlmeier S. The pandemic of physical inactivity: global action for public health. Lancet 2012; 380(9838):294-305.

47. Guthold R, Stevens GA, Riley LM, Bull FC. Worldwide trends in insufficient physical activity from 2001 to 2016: a pooled analysis of 358 population-based surveys with 1.9 million participants. Lancet Glob Health 2018; 6(10):e1077-e1086.

48. Mielke GI, Hallal PC, Rodrigues GBA, Szwarcwald CL, Santos FV, Malta DC. Physical activity and television viewing among Brazilian adults: National Health Survey 2013. Epidemiol. Serv. Saúde 2015; 24(2):277-286.

49. Sá GBAR, Dornelles GC, Cruz KG, Amorim RCA, Andrade SSCA, Oliveira TP, Silva MMA, Malta DC, Souza MFM. The Health Academy Program as a strategy to promote health and healthy lifestyles: the national implementation scenario. Cien Saude Colet 2016; 21(6):1849-1860.

50. Fernandes AP, Andrade ACS, Costa DAC, Dias MAS, Malta DC, Caiaffa WT. Health Academies Program and the promotion of physical activity in the city: the experience of Belo Horizonte, Minas Gerais state, Brazil. Cien Saude Colet 2017; 22(12):3903-3914.

51. Tekin E, McClellan C, Minyard KJ. Health and Health Behaviors during the Worst of Times: Evidence from the Great Recession. IZA Discussion Paper 2013; 7538:1-48.

52. Nandi A, Charters TJ, Strumpf EC, Heymann J, Harper S. Economic conditions and health behaviours during the 'Great Recession'. J Epidemiol Commun $H$ 2013; 67(12):1038-1046. 
53. Machado IE, Monteiro MG, Monteiro RA, Lana FCF, Gawryszewski VP, Malta DC. Trends in mortality rates where alcohol was a necessary cause of death in Brazil, 2000-2013. Rev Panam Salud Publica 2018; 42:e9.

54. Global Burden of Disease (GBD) 2016 Alcohol Collaborators. Alcohol use and burden for 195 countries and territories, 1990-2016: a systematic analysis for the Global Burden of Disease Study 2016. Lancet 2018; 392(10152):1015-1035.

55. Garcia LP, Freitas LRS. Heavy drinking in Brazil: results from the 2013 National Health Survey. Epidemiol. Serv. Saúde 2015; 24(2):227-237.

56. Munhoz TN, Santos IS, Nunes BP, Mola CL, Silva ICM, Alicia M. Tendências de consumo abusivo de álcool nas capitais brasileiras entre os anos de 2006 a 2013: análise das informações do VIGITEL. Cad Saude Publica 2017; 33(7):e00104516.

57. Frone MR. The Great Recession and employee alcohol use: a U.S. population study. Psychol Addict Behav 2016 Mar; 30(2):158-167.

58. de Goeij MC, Suhrcke M, Toffolutti V, van de Mheen D, Schoenmakers TM, Kunst AE. How economic crises affect alcohol consumption and alcohol-related health problems: a realist systematic review. Soc Sci Med 2015; 131:131-146.

59. Brasil. Presidência da República. Lei No 9.294, de 15 de Julho de 1996. Dispõe sobre as restrições ao uso e à propaganda de produtos fumígeros, bebidas alcoólicas, medicamentos, terapias e defensivos agrícolas, nos termos do $\$ 4^{\circ}$ do art. 220 da Constituição Federal. Diário Oficial da União 1996; 17 jul.

60. Malta DC, Bernal RT, de Souza MF, Szwarcwald CL, Lima MG, Barros MB. Social inequalities in the prevalence of self-reported chronic non-communicable diseases in Brazil: national health survey 2013. Int J Equity Health 2016; 15(1):153.

61. Barros MBA, César Chester LG, Carandina L, Torre GD. Desigualdades sociais na prevalência de doenças crônicas no Brasil, PNAD-2003. Cien Saude Colet 2006; 1(4):11-926.

62. Francisco PMSB, Segri NJ, Barros MBA, Malta DC. Desigualdades sociodemográficas nos fatores de risco e proteção para doenças crônicas não transmissíveis: inquérito telefônico em Campinas, São Paulo. Epidemiol. Serv. Saúde 2015; 24(1):7-18.
63. Tavares NUL, Bertoldi AD, Mengue SS, Arrais PSD, Luiza VL, Oliveira MA, Ramos LR, Farias MR, Dal Pizzolet TS. Factors associated with low adherence to medicine treatment for chronic diseases in brazil. Rev Saude Publica 2016; 50(Supl. 2):1-11.

64. Gorski MT, Roberto CA. Public health policies to encourage healthy eating habits: recent perspectives. $J$ Healthc Leadersh 2015; 7:81-90.

65. Werneck GL, Carvalho MS. A pandemia de COVID-19 no Brasil: crônica de uma crise sanitária anunciada. Cad Saude Publica 2020; 36(5):e00068820.

66. Mendes LL, Campos SF, Malta DC, Bernal RTI, Sá NNB, Velásquez-Meléndez G. Validity and reliability of foods and beverages intake obtained by telephone survey in Belo Horizonte, Brazil. Rev. bras. epidemiol 2011; 14(Supl. 1):80-89.

67. Monteiro CA, Florindo AA, Claro RM, Moura EC. Validity of indicators of physical activity and sedentariness obtained by telephone survey. Rev Saude Publica 2008; 42(4):575-581.

68. Moreira AD, Claro RM, Felisbino-Mendes MS, Velasquez-Melendez G. Validity and reliability of a telephone survey of physical activity in Brazil. Rev. bras. Epidemiol 2017; 20(1):136-146.

Artigo apresentado em 13/11/2020

Aprovado em 13/11/2020

Versão final apresentada em 15/11/2020

Editores-chefes: Maria Cecília de Souza Minayo, Romeu Gomes, Antônio Augusto Moura da Silva 